\title{
Propuesta organizacional de un archivo de gestión para organizaciones empresariales
}

\author{
Irima Campillo Torres \\ Máster en Bibliotecología y Ciencias de la Información. Licenciada \\ en Educación en la Especialidad de Español - Literatura \\ Profesora da Universidad de Camaguey. Facultad de Informatica \\ Departamento de Bibliotecologia y Ciencias de la Información - \\ Havana, Cuba \\ E-mail: irima.campillo@reduc.edu.cu
}

\section{Idalmis Maria Cabrera Morales}

Master en Producción Avícola Sostenible. Ingeniera Pecuaria. Profesora da Universidad de Camaguey. Facultad de Informatica Departamento de Bibliotecologia y Ciencias de la Información Havana, Cuba

E-mail: idalmis.cabrera@reduc.edu.cu

\section{Resumen}

Hoy día la gestión eficaz de los recursos de información y documentación en las empresas, genera la búsqueda de soluciones prácticas y exitosas. El presente artículo aborda el papel fundamental que han tenido los archivos, desde la antigüedad hasta la actualidad y su influencia en las funciones que desarrollan las organizaciones empresariales, como elemento indispensable para su cambio continuo. El presente artículo proviene de una pesquisa realizada en la Empresa Constructora de Obras de Arquitectura e Industria, No 8 (ECOAI) del territorio camagüeyano. Propone la organización de un Archivo de Gestión con sus respectivas fases de: Análisis, Diseño e Implantación; cada una posee un plan de actuación especifico. La propuesta tiene en cuenta la interrelación existente entre fases y etapas con un enfoque sistémico, con la finalidad de organizar, conservar y difundir la documentación como parte estratégica del patrimonio documental. Las consideraciones finales son presentadas sobre la aplicación de la propuesta en el ambiente empresarial cubano.

\section{Palabras clave}

Documentos. Archivo de gestión. Organizaciones empresariales. Enfoque sistémico.

\author{
Organizational proposal of a file of \\ administration for managerial organizations
}

\begin{abstract}
Nowadays the effective administration of the resources of information and documentation in the enterprises generates the search of practical and successful solutions. The present article approaches the fundamental role that files have had, from the antiquity until the present time, and their influences on the functions that develop the managerial organizations, as an indispensable element for their continuous changes. It has been an investigation carried out in the Architecture and Industry Construction Enterprise 8 (ECOAI) of the Camagüey territory. It proposes the organization of a file of administration with their respective phases of: Analysis, Design and Implementation. Each one has a plan of specific performance. The proposal keeps in mind the existent interrelation between phases and stages based on a systemic focus for the purpose of organizing, preserving and diffusing the documentation as strategic part of the documental patrimony of the organization. The final considerations are presented on the application of the proposal in the Cuban managerial atmosphere.
\end{abstract}

\section{Keywords}

Documents. File of step. Entrepreneurial organizations. Systemic focus.

\section{INTRODUCCIÓN}

El tema de los archivos ha sido muy abordado en diversas literaturas especializadas al respecto; "los archivos son tan antiguos como la organización social de la humanidad", (MENA, 2005). Desde su surgimiento han estado al servicio de la sociedad imperante, para el control de actividades económicas, jurídicas y sociales en forma general.

Sin lugar a dudas, la materia prima de los archivos son los documentos, con su condición de fe pública, ellos son reflejo de las funciones y actividades del 
hombre, producto y testimonio de una gestión (DICCIONARIO DE TERMINOLOGÍA ARCHIVÍSTICA, 1992).

En tal sentido (OTLET citado por MOREIRO, 1998), refiere que documento es "toda fuente de información física que comunicase inscripciones, imágenes, sonido, texto, objetos con indicios de intervención humana, creaciones artísticas, e incluso materiales naturales".

Este concepto más amplio y universal sitúa al documento en el centro de un complejo proceso de comunicación y transmisión de conocimiento. Sus aportes convierten a la Documentación en otro antecedente de la Ciencia de la Información.

El surgimiento de la Ciencia de la Información fue concebido en un "contexto comunicativo y tecnológico", como repuesta de una nueva conceptualización para el estudio de los documentos. Hoy no puede entenderse la actividad documental lejos de estas técnicas novedosas, el aporte principal de los dispositivos tecnológicos en el análisis documental es decisivo y rápido al procesar grandes cantidades de informaciones y ponerlas a disposición de los usuarios, lo cual tiene como consecuencia la solución de problemas informativos y la toma de decisiones.

En tal sentido, los responsables de dirigir grandes y complejas organizaciones, dependen de la información para la obtención del éxito. Al respecto (VICKERY citado por MOREIRO, 1998), señala que "la información debe ser aceptada como un recurso que requiere administración adecuada, igual que el dinero, la mano de obra y los materiales".

Este postulado presupone que en cada organización alguien debe poseer la responsabilidad y autoridad para administrar los recursos de información y estar adecuadamente preparado para hacerlo. El servicio de documentación de una empresa actúa como puente entre el mundo de la información y las necesidades de los usuarios de documentación en la empresa.
Sin lugar a dudas "desde la última década del pasado siglo las organizaciones ganaron conciencia de la necesidad de obtener ventajas con relación a sus competidores e identificaron que las mayores ventajas se obtenían de aquellos elementos que se derivaban del quehacer de sus trabajadores y técnicos, y que principalmente el conocimiento constituía el aspecto que marcaría diferencias en el futuro" (PONJUÁN, 1998).

Ante este escenario que demanda la planificación coordinación y control de la información en forma ordenada, para aumentar los beneficios y mantener la competitividad en las empresas, se establecen políticas de prioridad relacionadas con estas acciones.

\section{LOS ARCHIVOS DE LAS EMPRESAS EN LA SOCIEDAD ACTUAL}

Con el decursar del tiempo la actividad archivística se perfecciona y se establecen regulaciones que permiten un sustancial avance en el control y preservación de los documentos y las unidades administrativas que los contienen, lo que garantiza una mejor conservación del patrimonio documental.

Es preciso señalar que con independencia de la etapa en que se encuentre un documento, es innegable el valor que representa en todos los casos, por ello, se coincide con lo planteado por (BARATA, 2004), al referir que "la gerencia y preservación a largo plazo de los documentos, es una preocupación principal concerniente a los archivos, pues "conservar los documentos, implica diversos retos incluyendo, por supuesto una política de conservación"

El crecimiento a menudo, caótico y desordenado, de las informaciones que contienen los documentos y su incidencia en la vida social, económica y cultural ha originado la evolución hacia la Sociedad de la Información. Las Tecnologías de Información y Comunicación (TICs), permiten un aumento significativo de la cantidad y calidad de las 
informaciones y promueven cambios sustanciales en la cultura ciudadana que emanan una mejora de la competitividad y, sobre todo, de la calidad de vida.

El reconocimiento del papel que pueden tener los archivos en el marco de la denominada Sociedad de la Información, es un hecho aún bastante reciente que se empieza a tomar en consideración, junto con el esfuerzo desplegado por el colectivo profesional de la gestión de documentos; la introducción de las TICs en el tratamiento documental, facilita el acceso de los ciudadanos a la información y promueve mejoras efectivas en la formación de los profesionales.

El nuevo milenio globalizado, establece que el desarrollo empresarial alcance un gran protagonismo, se buscan nuevas formas organizativas que permitan gestionar la información contenida en los documentos; "una gestión eficiente de documentos debe "articularse con las TICs, no solo para garantizar la transparencia, el acceso a la información y la rendición de cuentas, sino también para maximizar el uso de la información presente y futura". (REVAH, 2009).

Es válido reconocer que muchas empresas cambian su orientación hacia enfoques innovadores que integren y complementen las capacidades existentes y a la vez permitan crear nuevas estructuras. Este nuevo contexto impone a las organizaciones, la necesidad vital de disponer de adecuadas infraestructuras de comunicación y de sistemas de información, que les permitan obtener los datos necesarios a fin de conseguir un conocimiento real y suficiente del entorno, y que por supuesto involucre los procesos de planificación y toma de decisiones.

En el ambiente empresarial, cada día es más común la incertidumbre de sus directivos para la toma de decisiones adecuadas y que sean proporcionales en eficiencia y eficacia. En las propias organizaciones existe una dispersión de datos e información que, en ocasiones, duplica tareas y hace imposible su recuperación por parte de quienes la necesitan en el momento oportuno; un buen número de empresas presentan estructuras obsoletas e inadecuadas para atender, con suficiencia los requerimientos actuales.

Precisamente el control de los documentos desde el momento de su producción en las oficinas, es la condición indispensable para poder recuperarlos tanto en su calidad de testimonio jurídico-administrativo como de elemento integrante del patrimonio histórico. Este control, exige una colaboración estrecha y coordinada entre las oficinas y los archivos de cada departamento.

El archivo de gestión es dentro del ciclo vital de los documentos, "el archivo de la oficina que reúne su documentación en trámite o sometida a continua utilización y consultas administrativas por las oficinas" (DICCIONARIO DE TERMINOLOGÍA ARCHIVÍSTICA, 1992), "es un archivo vigente, activo, donde surgen los documentos y también donde se archiva toda la documentación recibida; por su frecuente consulta y utilización es imprescindible que haya una perfecta organización, pues ello garantiza la agilidad en el trámite y la eficiencia del trabajo de la misma". (FERNÁNDEZ, 1999).

El plazo establecido para la transferencia de la documentación de los archivos de gestión u oficina al archivo central, según la normativa es de cinco años,- cada país debe establecer y regular los plazos de transferencia. El objetivo esencial del archivo de gestión es la organización de la documentación que se encuentra en continua formación, para atender las necesidades de información de las propias oficinas que producen los documentos, las necesidades de la organización en su conjunto y los requerimientos del Sistema Archivístico Institucional (SAI).

Existe cierto consenso en la literatura que aborda la organización del archivo de gestión. Según (NÚÑEZ, 1999), establece un conjunto de pasos fundamentales en el tratamiento documental:

1. Identificación y valoración del fondo documental; 
2. Elaboración del cuadro de clasificación y catálogo de tipos de documentales de la oficina;

3. Clasificación del fondo y de los documentos;

4. Ordenación de los documentos dentro de cada serie documental;

5. Descripción de unidades documentales y elaboración de los instrumentos de consulta de fondo;

6. Instalación del fondo en el depósito de archivo.

La práctica revela que hoy día las empresas que poseen archivos de gestión son más eficientes en el uso y manejo de la documentación, por lo que se necesita de acciones encaminadas a asegurar y facilitar el acceso a los documentos y no sólo recuperarlos como objetos individuales, sino también encontrar los que son verídicos y fiables, los que tienen relación entre ellos y los que se han generado dentro de una actividad específica, "y no bastan las soluciones tradicionales, sino que se precisan planes corporativos de gestión de documentos que respondan a una concepción global y a una implantación". (BUSTELO, 2000).

En tal sentido se plantea que las organizaciones empresariales cubanas se encuentran inmersas en un proceso de cambio continuo denominado Perfeccionamiento Empresarial (Bases del Perfeccionamiento Empresarial, 2007), el cual tiene como objetivo fundamental incrementar al máximo la eficiencia y la competitividad en la empresa; para el logro de este propósito, se precisa la existencia de una adecuada gestión de información científico tecnológica, que implique, necesariamente una correcta gestión de recursos de información, determinación de los costos de la misma y asignación de valores que se deriven del manejo de la información". (GARCÍA, 2004).

Las organizaciones que se encuentran en Perfeccionamiento Empresarial deben tener creados sus propios archivos de gestión u oficina, con las funciones previamente establecidas en los Lineamientos del Decreto Ley 265, 2009.

La propuesta de archivo de gestión que se presenta en esta investigación, se realizó a partir de una revisión exhaustiva de la documentación existente en empresas cubanas de la construcción, específicamente, en la ECOAI No 8 de Camagüey, con la finalidad de conocer los caracteres internos y externos de los documentos y los tipos de documentos que se generan como reflejo del actuar de la entidad.

\section{FASES Y ETAPAS PARA LA ORGANIZACIÓN DEL ARCHIVO DE GESTIÓN}

\section{FASE: ANÁLISIS.}

Etapa I: Análisis estratégico de la empresa

En esta primera etapa participan los directivos, responsables de las oficinas de la empresa y personal seleccionado para la administración del archivo en cuestión.

Plan de actuación:

En etapa además del análisis estratégico de la empresa, se tienen en cuenta también los problemas más comunes afectan la organización de un archivo de gestión, tales como:

- Acumulación de documentos (existencia de documentos duplicados y obsoletos).

- Redefinición de tareas encomendadas en cada una de las áreas y direcciones de la empresa.

- Diferentes criterios de archivo entre el personal de la empresa.

- Falta de personal calificado. (Proyecciones)

- Mobiliario y material incorrecto para archivar los documentos

- Insuficiente utilización de los documentos digitales en relación con los documentos en soporte manual. 
Etapa 2: Estudio de la base legal de la empresa y los procedimientos para su creación.

Objetivo: Conocer la legislación vigente que afecta la empresa en cuestión así como el uso y manejo de la documentación y otras fuentes que faciliten el conocimiento de las direcciones y los métodos de archivos.

Plan de actuación:

Los documentos necesarios a estudiar esta etapa:

- Legislación que afecta a cada dirección en particular;

- Expediente de Perfeccionamiento Empresarial. (Caracterización general de la empresa);

- Reglamentos, Resoluciones, Cartas, Circulares de cada dirección;

- Normas de procedimientos elaboradas para la tramitación de los diferentes asuntos;

- Estudio del organigrama de la entidad;

- Informe de los responsables de las direcciones de la empresa;

- Estudio de manuales sobre archivística.

\section{FASE: DISEÑO}

Etapa I: Esta etapa comienza con un análisis general de la situación actual de los documentos que tiene la organización, con la finalidad diseñar el archivo de gestión, acorde a las características y a las funciones de cada oficina, para garantizar que los procesos se lleven a cabo bajo condiciones controladas.

Plan de actuación:

Este análisis se lleva a cabo mediante contacto directo con los directivos de la empresa y a través de reuniones con las direcciones departamentales hasta obtener una visión global de la situación actual de la empresa y condiciones reales de las oficinas.
- Definición de los tipos documentales en que se plasman las funciones de la organización;

- Revisión de los documentos de forma general para conocer cuáles son afines a las funciones que se desarrollan en la organización;

- Elaboración del cuadro de clasificación;

- Definición de las series documentales;

- Determinación de los plazos de transferencias de los documentos a los diferentes archivos del sistema;

- Definición el nivel de acceso a los documentos;

- Gestión y control de expedientes;

- Normalización de formularios;

- Racionalización de los procedimientos;

- Definición de las políticas de conservación, eliminación, almacenamiento de la documentación en cualquier soporte;

- Determinación de los depósitos de almacenamiento de la documentación;

- Definición de los criterios de organización de la documentación y pautas para su control y recuperación;

- Elaboración de un informe que recoja los requerimientos necesarios para el archivo de gestión por cada una de las oficinas implicadas.

Etapa 2: Definición de los soportes en que se plasmará cada tipo documental durante el proceso de generación de los documentos, con vista a su conservación y utilización.

Plan de actuación:

- Análisis del volumen de producción de documentos y la situación organizativa y tecnológica en la que se encuentra la empresa. 
- Análisis de la identificación de los recursos de información ya realizadas por cada una de las oficinas de la empresa (tipo de fuentes, servicios y sistemas de información).

- Definición del soporte en que se plasmará cada documento según las condiciones reales de cada oficina.

\section{FASE: IMPLEMENTACIÓN}

En esta fase las acciones de formación y motivación de los implicados en el nuevo concepto de archivos de gestión, deben completarse con una adecuada comunicación de los beneficios a obtener por el sistema. Este proceso requiere una estrecha colaboración entre los directivos organizativos de la empresa, los técnicos informáticos y el personal seleccionado para llevar adelante la creación de los archivos.

Plan de actuación:

- Elaboración de los procedimientos de trabajo y asignar responsabilidades en materia de gestión archivística.

- Establecimiento de una política adecuada de comunicación de objetivos del proyecto y de formación.

- Periodificar el proceso de implantación: planificar el día a día y tomar decisiones respecto a la documentación patrimonial de la empresa.

Para la organización de los documentos de una oficina todas las partes implicadas deben estar convencidas de la necesidad que requiere este proceso y un aspecto de especial atención es la clasificación de los documentos de apoyo informativo, los cuales se deben ordenar según la (s) función (es) que cumplen, su disposición ha de ser cómoda y accesible.
La persona encargada de la organización y control documental en los archivos de gestión, vela por la correcta formación de los expedientes y emplea recursos, métodos, modelos, instrumentos u otros mecanismos establecidos para tales efectos, con vista a evitar su pérdida o extravío.

Los archivos de gestión proponen los plazos de conservación de la documentación a su custodia, los que son aprobados posteriormente por la Comisión de Valoración Documental correspondiente.

\section{INTERRELACIÓN ENTRE LAS FASES Y ETAPAS CON ENFOQUE SISTÉMICO}

El enfoque sistémico aplicado al estudio de las organizaciones plantea una visión inter, multi y transdisciplinaria que ayuda a analizar a la organización de manera integral; además permite la identificación y comprensión de los problemas organizacionales con mayor claridad, sus múltiples causas y consecuencias.

Por consiguiente, el archivo de gestión en la empresa es visto como un subsistema del sistema mayor, que es el SAI. Como se ha abordado en varias literaturas que emprenden la temática, en los archivos de gestión, concierne la primera edad de los documentos, es donde se produce la formación de estos, y se agrupa toda la documentación en trámite o sometida a una continua utilización y consulta administrativa.

Se prevé que su organización sea a través fases y etapas, que se relacionan entre sí, lo que permite un análisis detallado de la información contenida en los documentos (Entrada), determinada por los procedimientos a realizar en plan de actuación. Estos procedimientos no pueden ser aislados, la integración entre ellos condiciona el desarrollo óptimo del archivo; una vez realizados y evaluados, se obtiene un resultado que conlleva a la retroalimentación del sistema y por último la recuperación de los documentos (Salida). 
De esta forma, se percibe la organización del archivo como un ente integrado, conformado por partes, con una estructura que se desenvuelve en un entorno determinado, se posee la capacidad de poder detectar con la amplitud requerida tanto la problemática, como los procesos de cambio manera integral. Se coincide con (ALBERCH, 2005), el cual plantea que "la propuesta de un sistema de gestión integral de documentos, precisa de la formulación de una nueva cultura administrativa y gerencial, que asuma con seriedad las ventajas y los costes de la organización".

\section{RESULTADOS ALCANZADOS CON LA APLICACIÓN DE LA PROPUESTA}

- Aumento de los niveles de eficiencia en la gestión empresarial;

- Utilización adecuada de la información en función de las actividades de la empresa;

- Cada una de las acciones a ejecutar en las diferentes etapas permitió el desarrollo de los procesos de gestión documental, gestión de información y la gestión del conocimiento, en estrecha relación con los elementos organizativos y tecnológicos de la ECOAI No 8;

- Incremento de la cultura organizacional de la empresa a través de la superación constante de los implicados en materia de archivos;

- Confección del Cuadro de Clasificación de la empresa;

- Conocimiento de legislaciones vigentes;

- Control de los documentos de la unidad administrativa.

\section{CONSIDERACIONES FINALES}

Es indudable el papel que desempeñan los archivos de gestión en las organizaciones empresariales, pues lleva implícito una serie de procesos enfocados hacia la realización de diversas actividades, entre las que se pueden destacar la identificación, manejo, evaluación y utilización de diferentes documentos, que se generan dentro de la empresa.

La propuesta constituye un importante instrumento para implementar acciones de esta índole en otras empresas, acorde a sus objetivos estratégicos, desarrolla la independencia de las mismas y permite índices de eficacia.

La implementación de la propuesta es un reto que trasciende cada día en el nuevo contexto empresarial, los cambios tecnológicos actuales permiten el desarrollo de organizaciones eficientes en la gestión de documentos, flexibles y aptas para alcanzar estadios superiores.

Específicamente en el caso de la ECOAI No 8, permitió la conservación del patrimonio documental de la empresa, acorde a los objetivos identificados.

Los resultados que se obtengan a partir de la aplicación en otras empresas, posibilitan un cambio de mentalidad que debe producirse en todos los implicados en el proceso de la gestión empresarial.

Artigo submetido em 29/08/2010 e aceito em 09/09/2011. 


\section{REFERENCIAS}

ALBERCH, R. Los sistemas de gestión de documentos como aliados de las politicas de eficiencia y rentabilidad en los ayuntamientos. Disponível em: $<$ http:/www.bcn.es/arxiu/publicaciones/castella/publicaciones. $\underline{\mathrm{htm}}>$. Acesso em: 3 may. 2005.

BARATA, K. Archives in the Digital Age. Journal of the Society of Archivists, v. 25, n. 1, p. 63-70, 2004.

Bases del Perfeccionamiento Empresarial. Disponível em: < http://www. nuevaempresa.cu >. Acesso em: 12 jun. 2009.

BUSTELO, C. Gestión documental en las empresas: una aproximación práctica. 2000. Disponível em: < http://www.inforarea.es/Documentos/ fesabid.pdf $>$. Acesso em: 3 abr. 2008.

CUBA. MINISTERIO DE JUSTICIA. Decreto Ley No. 265. Gaceta oficial de la República de Cuba. La Habana: Ministerio de Justicia, 2009 .

DICCIONARIO de terminología archivistica. Madrid: Dirección de Archivos Estatales, 1992.
FERNÁNDEZ, P. Manual de organización de archivos de gestión en las oficinas municipales. Granada: CEMCI, 1999.

GARCÍA, E. Gestión Documental en Intranet. Disponível em: < http:// www.aui.es/biblio/libros/expo98/gestion documental.htm $>$. Acesso em: 5 ago. 2008.

MOREIRO, J. A. Introducción al estudio de la información y la documentación. Antioquia: Universidad de Antioquia, 1998.

MENA, M. Gestión documental y organización de arcbivos. La Habana: Félix Varela, 2005.

NÚÑEZ, E. Organización y gestión de archivos. Gijón: Ediciones TRE, 1999.

PONJUÁN, G. Gestión de Información en las Organizaciones: Principios, conceptos y aplicaciones. Santiago de Chile: CECAPI, 1998.

REVAH LACOUTURE, M. La gestión documental en procesos estratégicos de mejora; un agente de oportunidad en una institución pública de Educación Superior: el caso de CIDE. Anales de la Documentación. v.12, p. 221- 233, 2009. 